\title{
USING PERCEPTUAL AND NEUROMUSCULAR RESPONSES TO \\ ESTIMATE MECHANICAL CHANGES DURING CONTINUOUS SETS IN THE BENCH PRESS
}

\author{
Mark Chapman ${ }^{1}$ \\ Eneko Larumbe-Zabala² \\ Mark Gosss-Sampson ${ }^{1}$ \\ N. Travis Triplett ${ }^{3}$ \\ Fernando Naclerio ${ }^{1}$
}

${ }^{1}$ Department of Life and Sports Science, University of Greenwich (UK)

${ }^{2}$ Clinical Research Institute, Texas Tech University HSC, Lubbock, TX (USA)

${ }^{3}$ Department of Health and Exercise Science Appalachian State University ASU Boone, NC (USA)

Corresponding author's full contact information:

Dr. Fernando Naclerio, PhD

Department of Life and Sport Science

University of Greenwich (UK)

Phone number: $+44(0) 2083318441$

E-mail: f.j.naclerio@gre.ac.uk 


\section{ABSTRACT}

The present study analyzed the effectiveness of the OMNI-RES (0-10) and the electromyographic signal for monitoring changes in the movement velocity during a set to muscular failure performed with different relative loads in the bench press exercise (BP). Ten males (30.8 \pm 5.7 years) were evaluated on eight separate days with 48 hours of rest between sessions. After determining the 1RM value, participants performed seven sets to failure with the following relative loads ranges: $30<40 \% ; 40<50 \%, 50<60 \%, 60 \%<70 \%, 70<80 \%$, $80<90 \%$ and $>90 \%$. The mean accelerative velocity (MAV), the Rating of Perceived Exertion (RPE) and the normalized root mean square (N-RMS) signal from the anterior deltoids were measured for every repetition of each set. The RPE expressed after the first repetition and when the maximum value of MAV was achieved over the sets was lower $(p<0.001, d>0.80)$ than the RPE associated with a 10\% drop in MAV and at failure. Furthermore, the initial RPE was useful to distinguish different loading zones between the light relative loads ( $30<40 \%$ vs. $40<50 \%$ vs. $50<60 \%$ ) and from these three zones to the higher relative load ranges (60 to $>90 \%$ ). Similar, but less clear, differences were observed for the N-RMS. In conclusion, apart from differentiating between relative loads, the RPE and in some cases N-RMS can both reflect changes associated with the initial, maximal, $10 \%$ drop in movement velocity and muscular failure during a continuous set in the BP.

KEYWORDS: OMNI-RES (0-10) scale, EMG, root mean square, muscular failure, accelerative velocity, resistance training. 


\section{INTRODUCTION}

The bench press is an integral part of the resistance training program utilized by most athletes to strengthen and gain upper body muscle mass (38). Many athletes in different disciplines use bench press on regular bases during the entire training season (39). For example, strong positive relationships have been reported between the bench press performance and playing ability in rugby players (3). Even though the maximal load that can be moved in one maximal repetition (1RM) has been traditionally considered a reference of athletes' upper body strength (7), the movement velocity achieved when moving a given relative load (\% 1RM) has also demonstrated to be a key component to control performance outcomes in resistance exercises (11). Along those lines Pareja-Blanco et al. (34) reported that using a lower volume resistance of training (-40\%), but maintaining higher movement velocities (80 to $100 \%$ of the maximum) resulted in inferior muscle mass gain, similar strength enhancement and superior improvements in vertical jump performance compared to training with longer sets and movement velocity loss of $40 \%$. Furthermore, regardless of the exercise, when training for power, a drop greater than $10 \%$ of the mechanical performance has been associated to a selective fatigue of fast motor units along with a progressive activation of the slow motor unit (12). Therefore, the importance of maintaining the exercise intensity avoiding undesired reductions in the movement velocity while performing resistance exercises could be considered of meaningful importance for strength and power athletes. Although devices such as accelerometers (10), linear transducers (31), or even recently developed iPhone app applications (6) are being currently using to monitor mechanical changes occurring during resistance exercises, from the practical point of view the availability of these devices could be difficult to implement on the day by day basis. Consequently athletes and coaches would need alternative methods to objectively monitor performance progression during resistance training.

The utility of rating of perceived exertion (RPE) scales to monitor changes in movement velocity using different relative loads have been previously demonstrated $(16,29$, 32). However, the association of the perceptual response and the concomitant muscular 
activation during resistance exercises is still unclear. Considering the increases in muscular activity are a direct result of motor efferent commands, which, in turn, cause an increase in the number of corollary signals toward the sensory cortex that may regulate the perception of effort (25), it could be expected that if the mechanical changes experienced during resistance exercise are anchored with the perceptual response they also should be reflected by concomitant change in the neuromuscular activity. Although some studies support the application of the perceptual response to reflect changes in the muscular activation after performing upper (26) and lower body (13) exercises, others suggested that the perceptual response is not capable of accurately detecting changes in motor unit activation during resistance exercises (28). Regardless of the aforementioned relationship between the electromyography (EMG) signal and the estimated perceptual response, researchers generally suggest that for controlling resistance training and the corresponding outcomes, monitoring movement velocity during resistance training is more relevant for coaches than establishing relationships between perceptual and neuromuscular responses (9, 32), Therefore, exploring how both the perceptual and the neuromuscular responses are associated with changes in movement velocity during resistance exercises would provide practical and useful information for controlling resistance exercises when athletes have no access to velocity control devices. To the best of our knowledge only Chapman et al (9) examined whether changes in the movement velocity during resistance exercises can be predicted by the perceived response and the level of muscular activation. Results from this study suggest that both OMNI-RES (0-10) and the neuromuscular activity can reflect fluctuations in movement velocity along a set to failure in the squat exercise.

The sensitivity of both the perceived exertion and the electromyographic signal to differentiate specific moments within the set where the movement velocity peaks, drops below certain levels (i.e., 10\%) from the maximum, or where the set approaches muscular failure still needs specific consideration for each particular exercise and athletic population. Consequently the aim of the present study was to explore the perception of effort and neuromuscular activity where the movement velocity peaks, decreases $10 \%$ with respect to 
the maximum, and at muscular failure during a continuous set, using different percentages of the $1 \mathrm{RM}$ in the free weight bench press (BP) exercise. Furthermore, the ability of the RPE and the neuromuscular activity to discriminate between relative loads across a wide range, from 30 to $100 \%$, divided into $10 \%$ incremental slots was also investigated. The available literature allows us to formulate the following hypotheses: 1) the RPE and the electromyographic signal at the end of the repetition will show significant differences between specific moments within the set where the velocity concomitantly reduced as the set approaches muscular failure; and 2) the RPE and the electromyographic signal measured at the beginning of each set will differentiate relative loads (as a percentage of $1 \mathrm{RM}$ ) utilized.

\section{METHODS}

Experimental Approach to the Problem

This study was designed to examine the applicability of the RPE and the electromyographic signal as methods for monitoring changes in the movement velocity during a continuous repetition set until muscular failure using different percentages of 1RM in BP. Furthermore, the use of both perceptual and electromyographic methods to differentiate relative loads and reflect differences in the movement velocity between light, moderate and heavy loads was also explored. After determining the individuals' 1RM values, participants were evaluated on seven occasions until achieving muscular failure with the following $1 \mathrm{RM}$ percentage ranges: 30 to $<40 \%$, 40 to $<50 \%$, 50 to $<60 \%, 60 \%$ to $<70 \%$, 70 to $<80 \%, 80$ to $<90 \%$ and $>90 \%$. The mean accelerative velocity (MAV), the OMNI-RES (0-10)

scale value, as a measure of the RPE, and the root mean square (RMS) surface electromyography signal (amplitude EMG) were obtained for all the repetitions of each set. The study assessed whether the instances where the movement velocity peaks, drops $10 \%$ from the maximum, or reaches muscular failure show different values of the subjective perception of effort and the neuromuscular activation measured over a set using seven percentage ranges (30 to $100 \%$ of $1 \mathrm{RM}$ ) in $\mathrm{BP}$. 


\section{Subjects}

Ten volunteers, recreationally resistance-trained males (age $30.8 \pm 5.7$ years, body mass $82.0 \pm 13.8 \mathrm{~kg}$, and height $178.8 \pm 5.20 \mathrm{~cm}$ ) took part in this study. To be eligible, participants had to be free of injury in the last three months prior to the intervention. They were furthermore required to train regularly between 2 to 3 times per week, using a routine including bench press exercises for a minimum of two and a maximum of 5 years before the start of the present study. Only recreationally trained individuals with no regular participation in other sports, including bodybuilding, power or weightlifting were recruited. Additionally, only individuals not having ingested ergogenic aids or any type of nutritional supplements affecting muscular performance 12 weeks or longer prior to the start of the study were eligible. Participants were instructed not to change their nutritional habits, and if any relevant change had been detected (i.e. becoming a vegetarian, restricting calories, taking nutritional supplements, etc.) participants’ data were excluded from the analysis.

All experimental procedures were conducted in accordance with the Declaration of Helsinki, and approved by the University Research Ethics Committee. All the participants were informed of the benefits and risks of the investigation prior to signing the institutionallyapproved informed consent document to participate in the study.

\section{Procedures}

Before the beginning of the study all the participants underwent two familiarization sessions. During these sessions, standard instructions, and RPE OMNI-RES (0-10) scale anchored procedures were explained to the participants in order to properly reflect the RPE for the whole body (35) after performing each singular repetition of the different resistance exercises including the BP.

The OMNI Perceived Exertion Scale for Resistance Exercise (OMNI-RES), developed and validated by Robertson and colleagues (35), includes both verbal and modespecific pictorial descriptors distributed along a comparatively narrow response range of 0 to 10 (Figure 1). These characteristics make the OMNI-RES scale a useful methodology to control the intensity of resistance exercises over other previously published scales. 


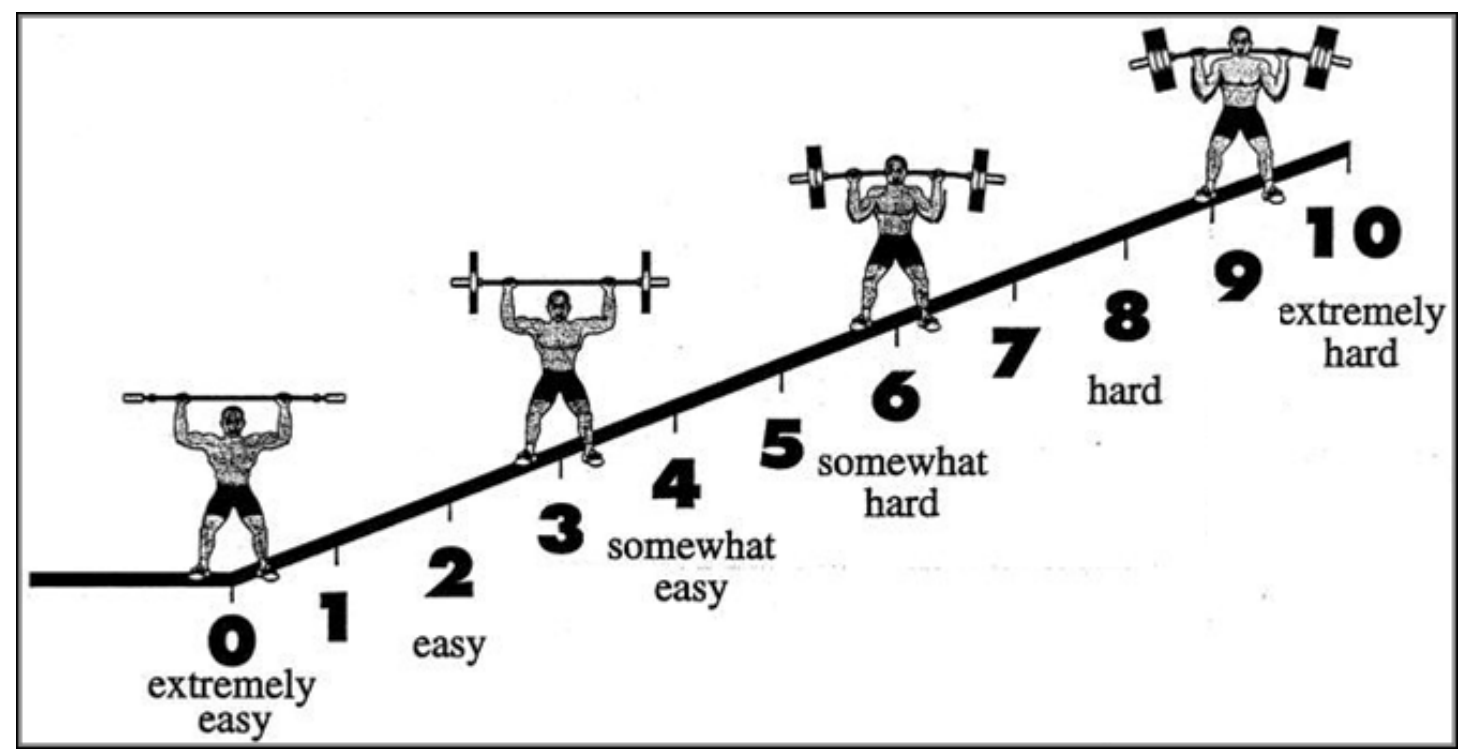

Figure 1. OMNI-RES (0-10) proposed by Robertson et al. (2003)

\section{Exercise}

The flat BP was performed using free weights. Participants were instructed to start the exercise lying down on a bench with their elbows fully extended and to lower the bar towards the chest using a controlled velocity. After a minimum pause (less than $1 \mathrm{~s}$.), participants performed the concentric phase with the maximal possible velocity. One qualified instructor (a certified strength and conditioning coach, CSCS or UK Strength and Conditioning Association) monitored the appropriate range of motion.

\section{Assessments}

$1 R M$ and Repetitions to Failure tests

The 1RM BP was determined in the first session. After $48 \mathrm{~h}$ rest and based on the 1RM results, participants performed seven-assessment sessions separated by 48 hours of rest. Each session comprised only one repetition to failure (RTF) test using the following 1RM percentages: $30 \% ; 40 \%$; $50 \% ; 60 \% ; 70 \% ; 80 \%$ and $90 \%$. As the availability of the free weight equipment (20 kg Olympic bar, 1.25, 2.5, 5, 10, 15 and $20 \mathrm{~kg}$ discs) did not always permit obtaining the exact amount of kg representing the aforementioned percentages, the nearest amount of $\mathrm{kg}$ provided it being equal or up to a maximum of $10 \%$ greater than the reference was considered for the test. Thus the following seven ranges were determined: 30 to 
$<40 \%, 40$ to $<50 \%, 50 \%$ to $<60 \%, 60$ to $<70 \%, 70$ to $<80 \%, 80$ to $<90 \%$ and $>90 \%$. In order to minimize the accumulated fatigue effect, sequencing of the RTF tests was randomized. Furthermore, participants were asked to abstain from any unaccustomed or hard exercise and refrain from caffeine intake, whilst maintaining similar sleeping hours and daily activities during the testing period. All the assessments were performed during the afternoon (12 to 6:00 pm).

\section{Measurement of movement velocity}

An optical rotary encoder (Winlaborat ${ }^{\circledR}$, Buenos Aires, Argentina, model WLEN01) with a minimum lower position register of $1 \mathrm{~mm}$ connected to the proprietary software Real Speed Version 4.20 was used for measuring the position and calculating the velocity (v) in $\mathrm{m} \cdot \mathrm{s}^{-1}$ achieved during each repetition of the BP. The cable of the encoder was connected to the right side of the bar in such a way that the exercise could be performed freely, which enabled the cable to move in either vertical direction of the movement (31). In order to avoid underestimation of the neuromuscular performance, the mean accelerative velocity (MAV) calculated from the accelerative portion of the concentric phase, during which the acceleration of the barbell was $\geq-9.81 \mathrm{~m} \cdot \mathrm{s}^{-2}$ was used for estimating changes in movement velocity (15).

The analysis of the MAV achieved during the RTF test was based on four specific events determined at: 1) the first repetition (MAV-1); 2) the repetition where the maximum value of MAV was achieved along the corresponding set (MAV-max); 3) the repetition where a drop of $10 \%$ in the MAV with respect to the MAV-max was identified (MAV-10\%); and 4) the MAV measured during the last repetition (MAV-F), just before the muscular failure on the last repetition of each set. As the maximum movement velocity is not always achieved during the first repetition, particularly when using light to submaximal loads (5) the velocity obtained at the first repetition and the repetition at which the maximal velocity was produced were independently analyzed. A $10 \%$ drop in the MAV was selected because a decline of such magnitude when performing explosive resistance exercises has been associated with 
selective fast twitch fiber's fatigue and a loss of movement speed which is not recommended for power development in athletes (21). The criterion analysis to determine the time point associated with the MAV-10\% was the performance of two continuous repetitions with a $10 \%$ reduction from the MAV-max. Furthermore, in order to determine the total drop in movement velocity compared the initial and the maximal velocity achieved within each set, the MAV-F was also considered (22).

Control of the rating of perceived exertion (RPE)

During the familiarization sessions and the RFT tests the participants were instructed to verbally report the RPE value indicating a number of the OMNI-RES (0-10) scale that reflects their overall muscular effort at the end of each repetition of the BP. The investigators used the same question before starting the first set of each exercise during the familiarization sessions and immediately prior to each of the seven RTF tests: "how hard do you feel your muscles are working during the exercise?” (33). A rating of 0 was associated with no effort (seating or resting), and a rating of 1 corresponded to the perception of effort while performing an extremely easy effort (27). A rating of 10 was considered to be maximal effort and associated with the most stressful exercise ever performed (24). The OMNI-RES (0-10) scale was in full view of participants at all times during the procedures.

\section{Electromyography data collection}

Bar position was monitored by 3D motion capture using $16 \mathrm{~mm}$ retro-reflective markers on each end of the bar. The sampling frequency of the motion capture was $200 \mathrm{~Hz}$ using 10 Oqus cameras (Qualisys, AB, Sweden). Synchronous to motion capture data surface EMG (DelsysBagnoli system, Boston, USA) was obtained via differential bipolar Ag-AgCl electrodes (10 mm center to center) placed on the anterior deltoid muscle of the dominant limb in accordance with SENIAM recommendations (19). Signal impedance and noise artifacts were minimized by skin preparation and taping of wires to prevent excessive movement during bench press. EMG signals were amplified (1 k gain) with a bandwidth of 20-450 Hz and a sampling frequency of $1000 \mathrm{~Hz}$. A single reference electrode was placed on the humerus. All synchronous data was acquired using Qualisys track manager software 
(Qualisys, AB, Sweden) and exported in .c3d format for post processing in Visual3Dtm (CMotion, Inc. Germantown, USA). EMG signals were corrected for zero offset by subtracting the signal mean, fully rectified and smoothed using a 2nd order bidirectional low pass filter with a cut-off frequency of $2 \mathrm{~Hz}$.

Data were collected throughout the entire RTF test for all the seven evaluated ranges. As the vertical displacement during the concentric phase (ascending movement) was recorded by the rotary encoder and time-synchronized with the EMG signal, only the EMG data relating to the concentric phase of each repetition was analyzed. As the present study was focused on identifying changes in the accelerative velocity at four specific times along each continuous set, the RMS signal was considered as the primary data for the analysis. The RMS value is the standard method for defining the effective amplitude of a time-varying, alternating signal, providing a meaningful representation of muscle activation at each of the analyzed time points (20). For normalization purposes, the muscle activity of the anterior deltoid was recorded and the maximum value determined during each of the RTF tests was considered the reference for normalizing the RMS signal (N-RMS) (8).

\section{Dependent Variables}

Three main dependent variables (MAV, RPE and N-RMS) were analyzed for each of the RTF tests. Furthermore, in order to assess the electromyographic signal and the perceived exertion to reflect changes in mechanical performance over a set to failure, the previously identified four consecutive time points for the MAV (MAV-1, MAV-max, MAV-10\% and MAV-F) were also used to determine the following corresponding values for the RPE and NRMS. RPE-1: OMNI-RES scale value of the first repetitions of each corresponding set; RPEmax: OMNI-RES scale value measured where the maximal mean accelerative velocity was measured for each corresponding set; RPE-10\%: OMNI-RES scale value measured when a $10 \%$ drop in maximal accelerative velocity was determined during corresponding set; RPE-F OMNI-RES scale value measured immediately after the end of each corresponding set. NRMS-1: Normalized signal achieved during the first repetitions of the corresponding set; $\mathrm{N}$ RMS-max: Normalized signal achieved during the repetition where the MAV was measured 
for each corresponding set; N-RMS-10\%: Normalized signal achieved during the repetition where a $10 \%$ drop of the MAV was determined for each corresponding set; N-RMS-F: Normalized signal determined for the last completed repetition for each corresponding set.

The test-retest reliability coefficients (ICCs) and standard error of measurement (SEM) for the seven RTF tests were $>0.92$ and between 0.13 to $0.02 \mathrm{~m}^{-1}$ or 0 to 1.8 considering the four times points measured at the MAV and the OMNI-RES (0-10) scale respectively $(9,32)$.

\section{Statistical Analyses}

Means and standard deviations (SD) were determined for all of the variables analyzed during the 1RM and RTF tests. Mauchly's Test of Sphericity was used for testing the normality of the difference data between all possible pairs of within-subject conditions. To analyze the existence of differences within a continuous set for each dependent variable (MAV, RPE, and N-EMG), one-way repeated measures analysis of variance (ANOVA) was applied for each of the seven tested data range (first hypothesis). Repeated measures ANOVAs were also performed to determine differences between time points (MAV-1, MAVmax, MAV-10\%, MAV-F) across the seven percentage ranges for each of the three dependent variables (MAV, RPE, and N-EMG) (second hypothesis). Bonferroni-adjusted post-hoc analyses were performed as appropriate for pairwise comparisons. Generalized eta squared $\left(\eta_{G}^{2}\right)$ and Cohen's $d$ values were reported to provide an estimate of standardized effect size (small d=0.2, $\eta_{G}^{2}=0.01$; moderate $\mathrm{d}=0.5, \eta_{G}^{2}=0.06$; and large $\mathrm{d}=0.8, \eta_{G}^{2}=0.14$ ). In order to provide useful information for controlling the load estimate, changes in movement velocity through the perception of effort the CIs (95\%) of the RPE variables were calculated. Average values are reported as mean \pm SD unless stated otherwise. Statistical power for the evaluations ranged from 0.85-1.00. The significance level was set at 0.05.

\section{RESULTS}

The $1 \mathrm{RM}$ mean value was $98.50 \pm 15.42 \mathrm{~kg}\left(1.22 \pm 0.20 \mathrm{~kg}\right.$. body weight $\left.^{-1}\right)$. The average relative load values and the total number of repetitions performed in each of the 
seven ranges were as follows: 1) $31.36 \pm 0.80 \%$ and $63.80 \pm 7.94$ repetitions; 2) $41.47 \pm 0.78 \%$ and $42.10 \pm 5.25$ repetitions; 3) $51.23 \pm 1.32 \%$ and $31.50 \pm 3.68$ repetitions; 4$) 61.38 \pm 0.75 \%$ and 23.70 \pm 2.71 repetitions; 5) $71.49 \pm 1.08 \%$ and $15.80 \pm 2.57$ repetitions; 6$) 81.45 \pm 0.91 \%$ and $10.9 \pm 1.96$ repetitions; 7) $91.63 \pm 0.95 \%$ and $4.60 \pm 1.50$ for 30 to $<40 \%, 40$ to $<50 \%, 50 \%$ to $<60 \%, 60$ to $<70 \%, 70$ to $<80 \%, 80$ to $<90 \%$ and $>90 \%$ respectively.

\section{Mean Accelerative Velocity (MAV)}

Table 1 shows the mean $\pm \mathrm{SD}$ of the MAV values, and the corresponding four time points analyzed along the RTF test within and across the seven ranges evaluated.

\section{Table 1}

\section{Comparison of the four time points within each range}

Significant main time effects were observed for the seven ranges. For the first 4 ranges (30 to $70 \%)$, significant differences and large effect sizes ( $d>0.80)$ were observed between the four time points with the exception of MAV-1 vs. MAV-10\%. For the three heaviest ranges (70 to $<90 \%)$ MAV-F was different to the other three times points. At 70 to $<80 \%$, both MAV-1 and MAV-max were different from the MAV-10\%.

Comparison of each of the time points across the ranges

Significant main range effects were observed for the four analyzed time points. Pairwise comparison revealed significantly higher MAV-1 and large effect sizes ( $d>0.80)$ for the first three ranges (30 to $<60 \%$ ) compared to other 4 ranges. Additionally, the MAV- 1 was higher at both 60 to $<70 \%$ and 70 to $<80 \%$ compared to $>90 \%$. The MAV-max and MAV$10 \%$ showed significantly lower values as the relative load increases across the seven assessed ranges (Table 1). No differences were observed between the MAV-F. Rating of Perceived Exertion (RPE)

Table 2 shows the mean \pm SD of the RPE values, and the corresponding four time points analyzed along the RTF test within and across the seven ranges evaluated.

\section{Table 2}


Significant main time effects were observed for the seven ranges. For the lightest (30$40 \%)$ and the three heaviest ranges (>70\%), both RPE-1 and RPE-max were similar and significantly lower ( $d>0.80$ ) than the RPE- $10 \%$ and RPE-F. For the 40 to $<50 \%$ RPE- 1 was different to RPE-10\% however RPE-max and RPE-10\% were similar. For the 50 to $<60 \%$ the RPE measured at the four time points were different between them $(d>0.80)$. The RPE-F was different from the other three time points at all the seven ranges $(\mathrm{d}=0.80)$.

Comparison of each of the time points across the ranges

Significant main range effects were observed for the RPE-1; RPE-max and RPE$10 \%$. The RPE-1 and RPE-max were lower ( $\mathrm{d}>0.8$ ) when compared the values expressed at the 30 to $40 \%$ and $>40$ to $50 \%$ to all the other 6 ranges. Furthermore, RPE- 1 and RPE-max at both $>60$ to $70 \%$ and $>70$ to $80 \%$ were lower ( $d>0.80$ ) than the observed at the two heaviest ranges $(>80 \%$ to $>90 \%)$.

The RPE-10\% was lower at the lightest range (30 to 40\%) compared to the other 6 ranges. Significant lower values were also observed between the $>40$ to $50 \%$ to the 4 heaviest ranges, from $>50$ to $60 \%$ to the three heaviest ranges and from $>70$ to $80 \%$ to the two superior ranges (Table 2). No differences were observed for RPE-F across the seven range percentages.

Table 3 depicts the 95\% CI limits for the four analyzed RPE variables. The RPE associated with each of the seven relative load ranges, could be used for selecting the load (RPE-1) and estimate changes in movement velocity (RPE-max and RPE-10\%) while performing continuous sets in the BP exercise.

\section{Table 3}

Amplitude EMG, Normalized root mean square signal (N-RMS)

Table 4 shows the mean \pm SD of the N-RMS values, and the corresponding four time points analyzed along the RTF test within and across the seven ranges evaluated.

Comparison of the four time points within each range: 
Significant main time effects were observed at $>40$ to $50 \%$; $>70 \%$ to 80 and $<90 \%$ ranges. No other main time effects were determined. Pairwise comparison revealed significant differences between the 4 time points only for the $>40$ to $50 \%$ range ( $d=0.80$ ). At $>50$ to $60 \%$ the N-RMS- 1 was lower than N-RMS-max and at $>60$ to $70 \%$ both N-RMS- 1 and N-RMS-max were lower the N-RMS-10\% whilst at $>70$ to $80 \%$ N-RMS-max was lower than N-RMS-10\% (d=0.80). Furthermore at the heaviest load (>90\%) both N-RMS-1 and NRMS-max showed lover values that N-RMS-F ( $\mathrm{d}=0.80)$. No other differences were observed (Table 4).

\section{Comparison of each of the time points across the ranges}

Significant main range effects were observed for the four analyzed variables.

Pairwise comparison revealed that the N-RMS-1 was significantly lower at 30 to $<40 \%$ compared to the other 6 ranges. Additionally, N-RMS- 1 was lower at $>40$ to $50 \%$ compared to the two heaviest ranges ( $d=0.80$ ), and from the $>50$ to 60 and $>60$ to 70 respect to the heaviest range $(\mathrm{d}=0.80)$.

The N-RMS-max and N-RMS-10\% showed significant lower values at both $30<40 \%$ and $40<50 \%$ with respect to the others 5 ranges. No other main range effects were observed.

\section{DISCUSSION}

The main finding of the present investigation was that the RPE measured by the OMNI-RES (0-10) scale is a valuable methodology to detect movement velocity fluctuations during a continuous set until volitional failure and to discriminate the relative load used (\% $1 \mathrm{RM})$ in the BP exercise. The methodology utilized measures the perception of effort at the end of each repetition during continuous sets to failure over a broad range of loads (30 to $>90 \%$ 1RM). In the line of previous investigations $(9,32)$, four crucial time points where analyzed: (i) the initial RPE-1 which was associated with the relative load used (ii) the perception expressed when the maximal movement velocity is achieved during the set (RPEmax), (iii) the perception expressed when a drop of $10 \%$ of the movement velocity is determined (RPE-10\%), and (iv) the perception associated with muscular failure (RPE-F). 
The RPE-1 and RPE-max were similar in all the evaluated ranges but different from RPE$10 \%$ and the RPE-F. Thus, controlling the RPE from the beginning and along a set performed with the maximal movement velocity would be an acceptable procedure to estimate the moment where a drop $10 \%$ occurs, and to monitor further changes in velocity when the sets approach to the end. As expected, the MAV decreased as the set progressed toward the muscular failure. One noteworthy finding was that from light to moderate loads ( $30 \%$ to $<70 \%$ ), the MAV-1 was similar to MAV-10\% and lower than MAV-max. Reasons for the observed lower values of MAV-1 may be related to the lack of specific previous neuromuscular preparation (9). Some individuals may need 2 to 3 repetitions before reaching the highest power production using submaximal resistance during exercises such as bench press (4) or jump squat (3). This capability entails a specific physical conditioning requiring specifically oriented training interventions (2) that was not particularly developed in our participants. Conversely, the significant decrease of the movement velocity measured when a $10 \%$ loss of the MAV was determined could be caused by selective fast motor unit disconnection mainly observed during continuous maximal velocity repetition sets (37). Results from the present investigation confirm previous findings supporting the suitability of the RPE for monitoring movement velocity changes during continuous sets of various resistance exercises $(27,30,32)$. However, different from the aforementioned studies that only investigated the association of the perceptual response with changes of the movement velocity as a mechanical measure of fatigue, we integrated the analysis of the neuromuscular response. Therefore, the present study covered three main areas: 1) mechanical by the movement velocity, 2) perceptual by the OMNI-RES (0-10) scale and 3) neuromuscular by the N-RMS. The N-RMS signal did not reach significant differences between the four time points measured for each $10 \%$ slots nor when compared between each individual time point (N-RMS-1; N-RMS-max; N-RMS-10\% and N-RMS-F) across the seven assessed ranges. Nonetheless, it is worth noting that the pattern of increase of the EMG signal within each singular set or between the seven tested percentage ranges reflects both the decrease of the MAV and the corresponding increase of the RPE. The observed progressive increase of the 
N-RMS signal in the present study is coherent with the traditional concept that the amplitude of EMG signals rises with the increment of the active motor units and their discharge rates (23, 36). Consequently as the set progresses toward muscular failure or the load increases, more motor units will be recruited determining a concomitant increase of the N-RMS (28).

The present results indicate that both N-RMS-1and RMS-10\% were significantly lower for the first two ranges $(30<50 \%)$ respect to the others. Nonetheless, in particular the N-RMS-1 displayed a similar pattern as observed for the RPE with significant lower values measured at light $(30<50 \%)$ and moderate $(<70 \%)$ to heavy $(<80 \%)$ ranges compared to the values observed at the highest load ( $\geq 80 \% 1 \mathrm{RM}$ ). The observed pattern of EMG responses agreed with the study of Hollander et al. (20) who suggested a rise of the normalized RMS signal as the contraction duration increases between 2, 3, 4 and 5 sec in the knee extension exercise. For the present study the duration of each repetition increases as the set progress or the load was progressively increased. In summary, the analysis within each range permits the acceptance of the first hypothesis supporting the ability of the RPE and in some way the NRMS to show changes in the movement velocity during continuous repetitions sets in the BP exercise.

The analysis across the ranges indicates that either the RPE-1 or the RPE-max are different between the first three ranges $(30 \%<60 \%$ of $1 \mathrm{RM})$ and from these light loading zone to the moderate to heavy zones $(60<90 \%)$. Furthermore the RPE- 1 was also able to differentiate moderate loads ( $60<70 \%$ ) from the heaviest ( $80>90 \%$ ). Consequently, using the RPE-1 would represent a good option for differentiating relative light $(30<40 ; 40<50$; 50 $<60 \% 1 \mathrm{RM}$ ); moderate (60 to $<70 \% 1 \mathrm{RM})$ and heavy $(\geq 70 \% 1 \mathrm{RM})$ loads.

The present results permit the acceptance of the second hypothesis of utilizing the RPE and in some way the neuromuscular signal to differentiate loading ranges based on $10 \%$ slot of 1RM percentages. The observed results are in line of previous studies with powerlifters $(17,18)$ and recreationally resistance-trained individuals $(9,32)$ supporting the use of the perceptual response as a suitable tool for prescribing relative load in resistance exercises, as an alternative to traditional methods such as percentage of 1RM. 
Similar to the present study, Naclerio et al (32) reported that relative loads in BP could be clearly differentiated between light, moderate and heavy loads by perceived exertion. However, the study by Naclerio and colleagues was unable to further discriminate between $10 \%$ increments of $1 \mathrm{RM}$ as was observed in the present investigation for the first four ranges ( $30<40 \%$ vs. $40<50 \%$ vs. $50<60 \%$ vs. $60<70 \%$ ); see Table 2 . Potential differences in the participants' resistance training background or the longer familiarization period (12 sessions vs. 8) using the OMNI-RES scale could explain the more accurate identification of lighter loading zones of our participants.

The present study is not without limitations. A limited number of male volunteers ( $\mathrm{n}=12$ ) were studied, participants were young, recreationally resistance-trained, performing the BP and familiarized with the use of OMNI-RES (0-10) scale for a minimum of 12 sessions before the assessment procedures. Therefore, the present results cannot be applied to other populations such as high performance athletes or other exercises and modalities, especially if there are relevant mechanical differences (i.e., a single joint exercise like arm curl, or cyclic total body exercises like running, swimming or cycling) or employ different muscle groups (i.e., lower body like leg extension), which have been shown to produce different effort perceptions at the same percentage and repetitions when compared to lower body exercises (14). Although similar perceptual responses and neurophysiological performance would be observed in women or elderly participants, further studies are warranted. On the other hand, it is worth highlighting that participants served as their own controls, reducing the variability, and the design involved randomization for assigning different load conditions. In conclusion, the functional linkage among three main effort markers: performance (MAV), perceptual (RPE) and neuromuscular (EMG response), during resistance exercises is confirmed. In addition, our results corroborate the use of the RPE expressed from the OMNI-RES (0-10) scale to both estimate the relative amount of the load and to monitor changes of the movement velocity during continuous set to muscular failure or upper body resistance exercises. 


\section{PRACTICAL APPLICATIONS}

From the practical point of view, the main contribution of the present investigation is to show the ability of the OMNI-RES (0-10) scale to estimate mechanical events occurring at different instances along a set performed to muscular failure with different percentages of the 1RM load in the BP exercise. The 95\% CIs for the four RPE-analyzed variables depicted in Table 3 can be used by coaches and athletes as an approach to distinguish different resistance loading zones by anchoring the RPE-1 to a given range of relative load (\% 1RM). Furthermore, the RPE-max and RPE-10\% can be used to identify specific moments over a set where the MAV-max and MAV-10\% are respectively produced. For example, to improve explosiveness, the following OMNI-RES (0-10) scale values can be used to identify the corresponding \%1RM ranges: 1 to 2 for $30<40 \% 1 \mathrm{RM} ; 1.5$ to 3 for $40<50 \% 1 \mathrm{RM}$; 3 to 5 for $50<60 \%$ and $60<70 \% 1 \mathrm{RM}$ and 4 to 6 for $70<80 \% 1 \mathrm{RM}$. Athletes will be instructed to perform the exercise with maximal possible movement velocity avoiding RPE values greater than $4,6,7$ or $>7$ when exercising with $30<40 \% 1 \mathrm{RM} ; 40<60 \%$ 1RM; $60<70 \% 1 \mathrm{RM}$ and $70<80 \% 1 \mathrm{RM}$, respectively. On the other hand, for strength oriented workout using $>80 \%$ 1RM (1) the suggested RPE-1 would be around 6 , for the $>80$ to $90 \% 1 \mathrm{RM}$ and $<8$ when using $>90 \% 1 \mathrm{RM}$. 


\section{REFERENCES}

1. ACSM Position Stand. Progression Models in Resistance training for healthy adults. Med Sci Sport Exerc 41: 687-708, 2009.

2. Baker D. Acute and long-term power responses to power training: Observations on the training of an elite power athlete. Strength Cond J 23: 4756., 2001.

3. Baker D. A series of studies on the training of High Intensity Muscle Power in Rugby League Football Player. J Strength Cond Res 15: 198-209., 2001.

4. Baker D, Nance S, and Moore M. The load that maximizes the average mechanical power output during explosive bench press throws in highly trained athletes. J Strength Cond Res 15: 20-24, 2001.

5. Baker D and Newton RU. Changes in power output across a high-repetition set of bench throws and jump squat in highly trained athletes. J Strength Cond Res 21: 1007-1011, 2007.

6. Balsalobre-Fernandez C, Marchante D, Munoz-Lopez M, and Jimenez SL. Validity and reliability of a novel iPhone app for the measurement of barbell velocity and 1RM on the bench-press exercise. J Sports Sci: 1-7, 2017.

7. Bird SP, Tarpenning KM, and Marino FE. Designing resistance training programmes to enhance muscular fitness: a review of the acute programme variables. Sports Med 35: 841-851, 2005.

8. Burden A. How should we normalize electromyograms obtained from healthy participants? What we have learned from over 25 years of research. $J$ Electromyogr Kinesiol 20: 1023-1035, 2010.

9. Chapman M, Larumbe-Zabala E, Gosss-Sampson M, Colpus M, Triplett NT, and Naclerio F. Perceptual, Mechanical And Electromyographic Responses To Different Relative Loads In The Parallel Squat. J Strength Cond Res, 2017.

10. Crewther BT, Kilduff LP, Cunningham DJ, Cook C, Owen N, and Yang GZ. Validating two systems for estimating force and power. Int J Sports Med 32: 254-258, 2011.

11. Davies TB, Kuang K, Orr R, Halaki M, and Hackett D. Effect of Movement Velocity During Resistance Training on Dynamic Muscular Strength: A Systematic Review and Meta-Analysis. Sports Med 47: 1603-1617, 2017.

12. Duchateau J, Semmler JG, and Enoka RM. Training adaptations in the behavior of human motor units. J Appl Physiol (1985) 101: 1766-1775, 2006.

13. Duncan MJ, Al-Nakeeb Y, and Scurr J. Perceived exertion is related to muscle activity during leg extension exercise. Res Sports Med 14: 179-189, 2006.

14. Faigenbaum A, Milliken LA, and Cloutier G. Perceived exertion during resistance exercise by children. Percept Mot Skills 98: 627-637, 2004. 
15. Gonzalez-Badillo JJ and Sanchez-Medina L. Movement velocity as a measure of loading intensity in resistance training. Int J Sports Med 31: 347-352, 2010.

16. Hardee JP, Lawrence MM, Utter AC, Triplett NT, Zwetsloot KA, and McBride JM. Effect of inter-repetition rest on ratings of perceived exertion during multiple sets of the power clean. Eur J Appl Physiol 112: 3141-3147, 2012.

17. Helms ER, Brown SR, Cross MR, Storey A, Cronin J, and Zourdos MC. SelfRated Accuracy of Rating of Perceived Exertion-Based Load Prescription in Powerlifters. J Strength Cond Res 31: 2938-2943, 2017.

18. Helms ER, Storey A, Cross MR, Brown SR, Lenetsky S, Ramsay H, Dillen C, and Zourdos MC. RPE and Velocity Relationships for the Back Squat, Bench Press, and Deadlift in Powerlifters. J Strength Cond Res 31: 292-297, 2017.

19. Hermens HJ, Freriks B, Disselhorst-Klug C, and Rau G. Development of recommendations for SEMG sensors and sensor placement procedures. $J$ Electromyogr Kinesiol 10: 361-374, 2000.

20. Hollander DB, Worley JR, Asoodeh M, Wakesa D, Magnuson M, Dantzler DK, Didier JJ, and Kraemer RR. A comparison of resistance exercise perceived exertion and muscle activation at varied submaximal durations, loads, and muscle actions. J Strength Cond Res, 2015.

21. Hori N, Newton RU, and Nosaka K. Weightlifting Exercise Enhance Athletic Performance That Requires High-Load Speed. Strength Cond J 27: 50-55, 2005.

22. Izquierdo M, Gonzalez-Badillo JJ, Häkkinen K, Ibáñez J, Kraemer WJ, Altadill A, Eslava J, and Gorostiaga EM. Effect of loading on unintentional lifting velocity declines during single sets of repetitions to failure during upper and lower extremity muscle actions. Int J Sports Med 29: 718-724, 2006.

23. Izquierdo M, Gonzalez-Izal M, Navarro-Amezqueta I, Calbet JA, Ibanez J, Malanda A, Mallor F, Hakkinen K, Kraemer WJ, and Gorostiaga EM. Effects of strength training on muscle fatigue mapping from surface EMG and blood metabolites. Med Sci Sports Exerc 43: 303-311, 2011.

24. Lagally KM, Amorose AJ, and Rock B. Selection of resistance exercise intensity using ratings of perceived exertion from the OMNI-RES. Percept Mot Skills 108: 573-586, 2009.

25. Lagally KM, McGaw ST, Young GT, Heather CM, and Thomas DQ. Rating of perceived Exertion and Muscle Activity During The Bench Press Exercise in Recreational And Novice Lifters. J Strength Cond Res 18: 359-364, 2004.

26. Lagally KM, Robertson KM, Gallagher KI, Goss FL, Jakicic JM, Lephart S, and Goospaster B. Perceived exertion, electromyography and blood lactate during acute bouts of resistance exercise. Med Sci Sport Exerc 34: 552-559, 2002. 
27. Lins-Filho ODL, Robertson RJ, Farah BQ, Rodrigues SLC, Cyrino ES, and Ritti-Dias RM. Effects of exercise intensity on rating of perceived exertion during a multiple-set resistance exercise session. J Strength Cond Res 26: 466-472, 2012.

28. Looney DP, Kraemer WJ, Joseph MF, Comstock BA, Denegar CR, Flanagan SD, Newton RU, Szivak TK, DuPont WH, Hooper DR, Hakkinen K, and Maresh CM. Electromyographical and Perceptual Responses to Different Resistance Intensities in a Squat Protocol: Does Performing Sets to Failure With Light Loads Produce the Same Activity? J Strength Cond Res 30: 792799, 2016.

29. Mayo X, Iglesias-Soler E, and Fernandez-Del-Olmo M. Effects of set configuration of resistance exercise on perceived exertion. Percept Mot Skills 119: 825-837, 2014.

30. Mayo X, Iglesias-Soler E, and Kingsley JD. Perceived exertion is affected by the submaximal set configuration used in resistance Exercise. J Strength Cond Res 101519/JSC0000000000001886, 2017.

31. Naclerio F and Larumbe-Zabala E. Loading Intensity Prediction by Velocity and the OMNI-RES 0-10 Scale in Bench Press. J Strength Cond Res 31: 323329, 2017.

32. Naclerio F, Rodríguez-Romo G, Barriopedro-Moro MI, Jimenez A, Alavar B, and Triplett NT. Control of resistance training intensiy by the omni perceived exertion scale. J Strength Cond Res 25: 1879-1888, 2011.

33. Pageaux B. Perception of effort in Exercise Science: Definition, measurement and perspectives. Eur J Sport Sci: 1-10, 2016.

34. Pareja-Blanco F, Rodriguez-Rosell D, Sanchez-Medina L, Sanchis-Moysi J, Dorado C, Mora-Custodio R, Yanez-Garcia JM, Morales-Alamo D, PerezSuarez I, Calbet JAL, and Gonzalez-Badillo JJ. Effects of velocity loss during resistance training on athletic performance, strength gains and muscle adaptations. Scand J Med Sci Sports 27: 724-735, 2017.

35. Robertson RJ, Goss FL, Rutkowski J, Lenz B, Dixon C, Timmer J, Frazee K, Dube J, and Andreacci J. Concurrent Validation of the OMNI Perceived Exertion Scale For Resistance Exercise. Med Sci Sport Exerc 35: 333-341, 2003.

36. Sale DG. Neural adaptation to resistance training. Med Sci Sports Exerc 20: S135-145, 1988.

37. Tidow G. Muscular adaptation induced by training and de training -a review of biopsy studies. New Studies in Athletics 10: 47-56, 1995.

38. Wilson GJ, Elliot BC, and Kerr GK. Bar path and force Profile Characteristics for maximal and submaximal loads in the Bench press. Int J Sports Biomech 5: 390-402, 1989. 
39. Wong PL, Chaouachi A, Chamari K, Dellal A, and Wisloff U. Effect of preseason concurrent muscular strength and high-intensity interval training in professional soccer players. J Strength Cond Res 24: 653-660, 2010. 
Mechanical changes estimated by perceived exertion and the EMG signal 23

\section{ACKNOWLEDGMENTS}

The authors thank Kelly Cooper for her help and support during the data collection.

Competing interests:

The present manuscript has been read and approved by all of the listed coauthors. All coauthors meet the guidelines of coauthor ship.

The results of the present study do not constitute endorsement by the NSCA. The authors have no conflicts of interest to disclose and no specific funding from grant agencies was obtained to support the present study. 


\section{TABLES}

Table 1. Mean (SD) for the MAV and the analyzed time points within the sets and across the seven ranges evaluated

\begin{tabular}{|c|c|c|c|c|c|c|c|c|}
\hline \multirow[t]{2}{*}{ Variables } & \multicolumn{7}{|c|}{ Percentage ranges } & \multirow{2}{*}{$\begin{array}{l}\text { One way ANOVA } \\
\text { (7 assessments) }\end{array}$} \\
\hline & $30-40 \%$ & $40<50 \%$ & $50<60 \%$ & $60<70 \%$ & $70<80 \%$ & $80<90 \%$ & $>90 \%$ & \\
\hline $\operatorname{MAV}\left(\mathrm{m} \cdot \mathrm{s}^{-1}\right)$ & $*$ & $*$ & $*$ & * & 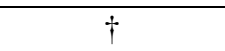 & $\ddagger$ & 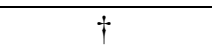 & \\
\hline MAV-1 & $0.71(0.14)^{a}$ & $0.65(0.11)^{a}$ & $0.58(0.09)^{a}$ & $0.53(0.08)^{b}$ & $0.46(0.05)^{c}$ & $0.34(0.12)$ & $0.27(0.03)$ & $\mathrm{F}(6,54)=36.65, \mathrm{p}=0.000, \eta_{G}^{2}=0.8$ \\
\hline MAV-max & $0.86(0.10)^{d}$ & $0.76(0.11)^{d}$ & $0.66(0.07)^{d}$ & $0.59(0.07)^{d}$ & $0.49(0.06)^{\mathrm{d}}$ & $0.39(0.05)^{d}$ & $0.27(0.03)^{d}$ & $\mathrm{~F}(6,54)=162.01, \mathrm{p}=0.000, \eta_{G}^{2}=0.9$ \\
\hline MAV-10\% & $0.77(0.09)^{d}$ & $0.68(0.10)^{d}$ & $0.59(0.08)^{d}$ & $0.52(0.07)^{d}$ & $0.43(0.05)^{d}$ & $0.34(0.06)^{d}$ & $0.21(0.04)^{d}$ & $\mathrm{~F}(6,54)=120.95, \mathrm{p}=0.000, \eta_{G}^{2}=0.93$ \\
\hline MAV-F & $0.21(0.08)$ & $0.21(0.08)$ & $0.15(0.05)$ & $0.16(0.05)$ & $0.15(0.05)$ & $0.16(0.07)$ & $0.12(0.03)$ & $\mathrm{F}(6,54)=2.72, \mathrm{p}=0.022, \eta_{G}^{2}=0.23$ \\
\hline $\begin{array}{l}\text { One way ANOVA } \\
\text { (4 time points) }\end{array}$ & $\begin{array}{c}\mathrm{F}(3,9)=283.68, \\
\mathrm{p}=0.000, \\
\eta_{G}^{2}=0.97\end{array}$ & $\begin{array}{c}\mathrm{F}(3,9)=97.08, \\
\mathrm{p}=0.000, \\
\eta_{G}^{2}=0.92\end{array}$ & $\begin{array}{c}\mathrm{F}(3,9)=223.69, \\
\mathrm{p}=0.000 \\
\eta_{G}^{2}=0.96\end{array}$ & $\begin{array}{c}\mathrm{F}(3,9)=191.51, \\
\mathrm{p}=0.000, \\
\eta_{G}^{2}=0.96\end{array}$ & $\begin{array}{c}\mathrm{F}(3,9)=127.81, \\
\mathrm{p}=0.000, \\
\eta_{G}^{2}=0.93\end{array}$ & $\begin{array}{c}\mathrm{F}(3,9)=14.42, \\
\mathrm{p}=0.000, \\
\eta_{G}^{2}=0.62\end{array}$ & $\begin{array}{c}\mathrm{F}(3,9)=59.98 \\
\mathrm{p}=0.000, \\
\eta_{G}^{2}=0.87\end{array}$ & \\
\hline
\end{tabular}

MAV: mean accelerative velocity; MAV-1: Maximal mean accelerative velocity achieved during the first repetitions; MAV-max: Maximal mean accelerative velocity;

MAX-10\%: Mean accelerative velocity measured when a 10\% decrease was determined; MAV-F: Mean accelerative velocity measured during the last repetition.

Differences within ranges: $* \mathrm{p}<0.001$ between the four time points with the exception of MAV-1 vs. MAV-10\%. $† p<0.05$ between the four time points with the exception of MAV-1 vs. MAV-max. $\ddagger \mathrm{p}<0.001$ between MAV-F vs. the other time points and from MAV-max to MAV-10\%.

Differences across ranges: ${ }^{\mathrm{a}} \mathrm{p}<0.001$ from the $>30-40$; $>40-50 \%$ and $>50-60 \%$ to the other ranges; ${ }^{\mathrm{b}} \mathrm{p}<0.05$ from $>60-70 \%$ to $>30-40$ and $>90 \%{ }^{\mathrm{c}} \mathrm{p}<0.05$ from $70-80 \%$ to $>90 \%$; ${ }^{\mathrm{d}} \mathrm{p}=<0.05$ between all the ranges with the exception of $>40-50 \%$ vs. $>50-60 \%$ and $>50-60 \%$ vs. $>60 \%$ to $70 \%$. 
Table 2. Mean (SD) for the RPE and the analyzed time points within the sets and across the seven ranges evaluated

\begin{tabular}{|c|c|c|c|c|c|c|c|c|}
\hline \multirow[t]{2}{*}{ Variables } & \multicolumn{7}{|c|}{ Percentage ranges } & \multirow{2}{*}{$\begin{array}{c}\text { One way ANOVA } \\
\text { (7 assessments) }\end{array}$} \\
\hline & $30<40 \%$ & $40<50 \%$ & $50<60 \%$ & $60<70 \%$ & $70<80 \%$ & $80<90 \%$ & $>90 \%$ & \\
\hline RPE (0-10) & * & * & $\dagger$ & $\ddagger$ & * & * & * & \\
\hline RPE-1 & $1.6(0.8)^{\mathrm{a}}$ & $2.3(1.16)^{\mathrm{a}}$ & $3.9(1.7)^{\mathrm{a}}$ & $3.7(1.6)^{b}$ & $5.0(1.3)^{b}$ & $6.3(0.9)$ & $8.0(0.5)$ & $\mathrm{F}(6,54)=68.4, \mathrm{p}=0.000, \eta_{G}^{2}=0.88$ \\
\hline RPE-max & $2.1(0.9)^{\mathrm{a}}$ & $3.2(0.9)^{\mathrm{a}}$ & $4.4(1.6)^{\mathrm{a}}$ & $4.9(0.9)^{\mathrm{b}}$ & $5.5(1.2)^{b}$ & $7.1(0.7)$ & $8.0(0.5)$ & $\mathrm{F}(6,54)=43.39, \mathrm{p}=0.000, \eta_{G}^{2}=0.83$ \\
\hline RPE-10\% & $3.4(1.5)^{\mathrm{c}}$ & $4.7(1.9)^{\mathrm{d}}$ & $5.1(1.3)^{\mathrm{e}}$ & $6.3(0.5)^{f}$ & $6.8(0.9)^{f}$ & $7.9(0.9)$ & $8.9(0.3)$ & $\mathrm{F}(6,54)=25.32, \mathrm{p}=0.000, \eta_{G}^{2}=0.74$ \\
\hline RPE-F & $10(0.0)$ & $10(0.0)$ & $10(0.0)$ & $10(0.0)$ & $10(0.0)$ & $10(0.0)$ & $10(0.0)$ & \\
\hline $\begin{array}{l}\text { One way ANOVA } \\
\text { (4 time points) }\end{array}$ & $\begin{array}{c}\mathrm{F}(3,9)=204.68, \\
\mathrm{p}=0.000, \\
\eta_{G}^{2}=0.96\end{array}$ & $\begin{array}{c}\mathrm{F}(3,9)=91.83, \\
\mathrm{p}=0.000, \\
\eta_{G}^{2}=0.91\end{array}$ & $\begin{array}{c}\mathrm{F}(3,9)=108.78, \\
\mathrm{p}=0.000, \\
\eta_{G}^{2}=0.92\end{array}$ & $\begin{array}{c}\mathrm{F}(3,9)=135.45, \\
\mathrm{p}=0.000 \\
\eta_{G}^{2}=0.94\end{array}$ & $\begin{array}{c}\mathrm{F}(3,9)=90.55, \\
\mathrm{p}=0.000, \\
\eta_{G}^{2}=0.91\end{array}$ & $\begin{array}{c}\mathrm{F}(3,9)=51.06, \\
\mathrm{p}=0.000, \\
\eta_{G}^{2}=0.85\end{array}$ & $\begin{array}{c}\mathrm{F}(3,9)=91.09, \\
\mathrm{p}=0.000, \\
\eta_{G}^{2}=0.91\end{array}$ & \\
\hline
\end{tabular}

RPE: rate of perceived exertion from OMNI-RES (0-10) scale; RPE-1: RPE value expressed after the first repetitions; RPE-max: RPE expressed after performing the repetition that produced the maximal mean acceleration velocity; RPE-10\%: RPE value measured when a 10\% drop in maximal accelerative velocity was determined; RPEF: RPE value expressed immediately after completed the set.

Differences within ranges: * $\mathrm{p}<0.05$ from RPE- 1 and RPE-max to RPE-10\% and RPE-F and between RPE- $10 \%$ and RPE-F, $\dagger \mathrm{p}<0.001$ RPE- 1 to RPE-10\% and from RPE-F to the other 3 time points, $\ddagger \mathrm{p}<0.05$ between the four time points.

Differences across ranges: ${ }^{\mathrm{a}} \mathrm{p}<0.001$ between the first three ranges $30-40$ vs. $>40$ to $50 \%$ vs. $>50$ to 60 , and from these three ranges to the other 4 superior ranges, ${ }^{b} \mathrm{p}<0.001$ to the two heaviest ranges 80 to $>90 \%$, ${ }^{\mathrm{c}} \mathrm{p}<0.05$ to the other 6 ranges ${ }^{\mathrm{d}} \mathrm{p}<0.05$ to the 4 heaviest ranges (60 to $>90 \%$ ) ${ }^{\mathrm{e}} \mathrm{p}<0.05$ to the 3 heaviest ranges $\left(70\right.$ to $>90 \%$ ) ${ }^{\mathrm{f}} \mathrm{p}<0.05$ to the two superior ranges ( 80 to $>90 \%$ ). 
Table 3. Mean CI (95\%) determined on the RPE main variables determined along the seven-repetition to failure test.

\begin{tabular}{|l|c|c|c|c|c|c|c|c|}
\hline \multirow{2}{*}{1 RM ranges } & \multicolumn{2}{|c|}{ RPE-1 } & \multicolumn{2}{c|}{ RPE-max } & \multicolumn{2}{c|}{ RPE-10\% } & \multicolumn{2}{c|}{ RPE-F } \\
\cline { 2 - 10 } & Lower & Upper & Lower & Upper & Lower & Upper & Lower & Upper \\
\hline 30 to $<40 \%$ & 1.1 & 2.1 & 1.6 & 2.6 & 2.5 & 4.3 & 10 & 10 \\
\hline 40 to $<50 \%$ & 1.6 & 3.0 & 2.7 & 3.7 & 3.5 & 5.9 & 10 & 10 \\
\hline 50 to $<60 \%$ & 2.9 & 4.9 & 3.5 & 5.3 & 4.3 & 5.9 & 10 & 10 \\
\hline 60 to $<70 \%$ & 2.7 & 4.7 & 4.4 & 5.4 & 6.0 & 6.6 & 10 & 10 \\
\hline 70 to $<80 \%$ & 4.2 & 5.8 & 4.8 & 6.2 & 6.2 & 7.4 & 10 & 10 \\
\hline 80 to $<90 \%$ & 5.7 & 6.9 & 6.7 & 7.5 & 7.4 & 8.4 & 10 & 10 \\
\hline$>90 \%$ & 7.7 & 8.3 & 7.7 & 8.3 & 8.7 & 9.1 & 10 & 10 \\
\hline
\end{tabular}

RPE-1: indicates OMNI-RES scale value determined after doing the first repetition of each repetition to failure test. RPE-max: indicates OMNI-RES scale value of the repetition where the maximal mean accelerative velocity was reached in each repetition to failure test. RPE-10\%: indicates OMNI-RES scale value expressed when a $10 \%$ decrease in the mean accelerative velocity was determined along each repetition to failure test. RPE-F: indicates the OMNI-RES scale value expressed after performing the last repetition of each ach repetition to failure test. 
Table 4. Mean (SD) for the N-RMS and the analyzed time points within the sets and across the seven ranges evaluated

\begin{tabular}{|c|c|c|c|c|c|c|c|c|}
\hline \multirow[t]{2}{*}{ Variables } & \multicolumn{7}{|c|}{ Percentage ranges } & \multirow{2}{*}{$\begin{array}{l}\text { One way ANOVA } \\
\text { (7 assessments) }\end{array}$} \\
\hline & $30-40 \%$ & $40<50 \%$ & $50<60 \%$ & $60<70 \%$ & $70<80 \%$ & $80<90 \%$ & $>90 \%$ & \\
\hline N-RMS (\%) & & $*$ & $\dagger$ & $\ddagger$ & $\S$ & & $\#$ & \\
\hline N-RMS-1 & $45.35(18.61)^{\mathrm{a}}$ & $55.32(15.27)^{\mathbf{b}}$ & $65.46(14.23)^{\mathrm{c}}$ & $67.65(16.40)^{\mathrm{c}}$ & $74.69(13.54)^{c}$ & $80.41(12.88)$ & $87.17(8.66)$ & $\mathrm{F}(6,54)=13.87, \mathrm{p}=0.000, \eta_{G}^{2}=0.61$ \\
\hline N-RMS-max & $54.97(15.58)^{\mathrm{e}}$ & $63.58(12.24)^{\mathrm{e}}$ & $77.17(9.50)$ & $79.30(6.71)$ & $79.90(8.45)$ & $83.15(12.07)$ & $87.17(6.66)$ & $\mathrm{F}(6,54)=15.96, \mathrm{p}=0.000, \eta_{G}^{2}=0.64$ \\
\hline N-RMS-10\% & $59.73(17.60)^{\mathrm{e}}$ & $68.60(13.02)^{\mathrm{e}}$ & $76.82(14.13)$ & $86.49(4.27)$ & $87.76(6.15)$ & $88.25(12.89)$ & $93.29(9.60)$ & $\mathrm{F}(6,54)=13.26, \mathrm{p}=0.000, \eta_{G}^{2}=0.6$ \\
\hline N-RMS-F & $75.81(22.03)$ & 83.96 (12.99) & 76.12 (14.99) & 78.31 (27.93) & 76.62 (17.38) & $82.94(10.44)$ & $97.23(5.21)$ & $\mathrm{F}(6,54)=2.64, \mathrm{p}=0.025, \eta_{G}^{2}=0.23$ \\
\hline $\begin{array}{l}\text { One way ANOVA } \\
\text { (4 time points) }\end{array}$ & $\begin{array}{c}\mathrm{F}(3,9)=4.28, \\
\mathrm{p}=0.140, \\
\eta_{G}^{2}=0.32\end{array}$ & $\begin{array}{c}\mathrm{F}(3,9)=16.15 \\
\mathrm{p}=0.000 \\
\eta_{G}^{2}=0.64\end{array}$ & $\begin{array}{c}\mathrm{F}(3,9)=1.78, \\
\mathrm{p}=0.175, \\
\eta_{G}^{2}=0.17\end{array}$ & $\begin{array}{c}\mathrm{F}(3,9)=2.47 \\
\mathrm{p}=0.084 \\
\eta_{G}^{2}=0.22\end{array}$ & $\begin{array}{c}\mathrm{F}(3,9)=3.83 \\
\mathrm{p}=0.021 \\
\eta_{G}^{2}=0.30\end{array}$ & $\begin{array}{c}\mathrm{F}(3,9)=0.88, \\
\mathrm{p}=0.463 \\
\eta_{G}^{2}=0.09\end{array}$ & $\begin{array}{c}\mathrm{F}(3,9)=9.54, \\
\mathrm{p}=0.000, \\
\eta_{G}^{2}=0.52\end{array}$ & \\
\hline
\end{tabular}

N-RMS: normalized room mean square signal, N-RMS-1: normalized signal achieved during the first repetitions; N-RMS-max: Normalized signal achieved during the repetition where the MAV was measured; N-RMS-10\%: Normalized signal achieved during the repetition where a 10\% drop of the MAV was determined; N-RMS-F: Normalized signal determined for the last repetition.

Differences within ranges: ${ }^{*} \mathrm{p}<0.05$ between the 4 time points, $\dagger \mathrm{p}<0.05$ from N-RMS- 1 to N-RMS-max, $\ddagger \mathrm{p}<0.05$ from N-RMS- 1 and N-RMS-max to N-RMS- $10 \%$, $\S$ $\mathrm{p}<0.05$ from N-RMS-max to N-RMS-10\%, \#p $<0.05$ from N-RMS-1 and N-RMS-max to N-RMS-F

Differences across ranges: ${ }^{\mathrm{a}} \mathrm{p}<0.05$ to the other 6 ranges; ${ }^{\mathrm{b}} \mathrm{p}<0.01$ to the two heaviest ranges $\left(>80\right.$ to $>90 \%$ ) ${ }^{\mathrm{c}} \mathrm{p}<0.01$ to $>90 \%{ }^{\mathrm{e}} \mathrm{p}<0.05$ from $30<40 \%$ and $40<50 \%$ to the others 5 superior ranges. 